\title{
Experimental analysis of the unsteady flow inside a Wells turbine
}

\author{
Fabio Licheri ${ }^{1, *}$, Pierpaolo Puddu ${ }^{1}$, Tiziano Ghisu ${ }^{1}$, and Francesco Cambuli ${ }^{1}$ \\ ${ }^{1}$ Department of Mechanical, Chemical and Materials Engineering, University of Cagliari, Italy
}

\begin{abstract}
One of the most promising technologies for sea-wave energy conversion is the one based on the Oscillating Water Column (OWC) principle. The system is composed of two units, an open chamber that converts the sea water motion into an alternating air-flow, and a turbine driven by the latter. The alternating flow of air requires a turbine capable of maintaining the same direction of rotation. The Wells turbine represents the simplest and most reliable device for this purpose. It is a self-rectifying axial turbine characterized by a rotor with symmetric blades staggered at 90 degrees relative to the axis of rotation. The vast majority of experimental works on Wells turbines and OWC devices analyzed their performance from a global point of view, often under steady conditions, in order to evaluate the pressure drop through the rotor, the torque produced and thus the turbine efficiency. This paper presents an experimental analysis of the three-dimensional flow inside a Wells turbine which operates in a facility capable of reproducing the alternating air-flow typical of an OWC system. The investigation is based on local flow measurements using several probes in order to describe the non-stationary air-flow, both up- and down-stream of the rotor at different heights, along the span of the blade. The investigation, conducted on a high-solidity turbine, details the behavior of the flow field inside the machine, aiming to provide a detailed description that can guide the aerodynamic optimization of the entire system (chamber and turbine) for a better energy conversion.
\end{abstract}

\section{Introduction}

Ocean energy represents one the most attractive renewable sources in virtue of its high availability and predictability [1]. For this reason, several researches have been carried out during the last four decades, presenting different solutions to harvest wave energy and convert it into electrical energy. One of the most interesting technologies is the one based on the Oscillating Water Column (OWC) principle, due to its simplicity and its lower environmental impact, especially when the plants are located near- or on-shore [2]. The plants based on the OWC principle can be considered made of two main units: a chamber, partially submerged under the sea level, where the wave motion induces an alternative movement of the air trapped in the chamber, and a turbine, placed at the top of the chamber and driven by the air-flow.

Different types of turbines have been proposed and studied coupled to OWCs systems [3], but probably the simplest is the Wells turbine invented by Dr. A. A. Wells in the 1980s

\footnotetext{
*Corresponding author: fabio.licheri@unica.it
} 
[4]. Its self-rectifying characteristic, due to the symmetrical blade arrangement, allows the turbine to keep its rotational direction unchanged under a bi-directional air-flow, and makes it simple and reliable. A number of authors have studied Wells turbines both with numerical [5-8] and experimental [9-12] approaches.

The vast majority of experimental analyses conducted in laboratory environments have been carried out to characterize turbines' performance [13-15], i.e. output torque, pressure drop across the rotor and finally turbine efficiency. Global analyses have been generally used to compare different turbine geometries and control strategies in order to select solutions with optimal performance, often expressed in terms of maximum output torque or mean efficiency. While global performance metrics are important to compare the merits of different configurations, detailed flow analyses can provide a better understanding of the flow dynamics leading to improved configurations.

Local analyses have been typically conducted under steady flow, i.e. inside uni-directional wind tunnels, although non-stationary flow conditions would be more representative of the actual operating conditions of the machine.

This paper presents a local flow analysis for a Wells turbine coupled with an OWC simulator, housed in the Department of Mechanical, Chemical and Materials Engineering (DIMCM) at the University of Cagliari $[9,10]$. The experimental investigation has been conducted by means of aerodynamic pressure probes in order to reconstruct the non-stationary flow field near the turbine blades at different heights.

In the first part of this manuscript, experimental rig and instrumentation are presented with a detailed description of the aerodynamic probe used in the analyses. Then, the measurements at the turbine's inlet and outlet are presented; local and global parameters are also determined from measurements and presented in this section of the manuscript. The last part summarizes the investigation results.

\section{Methodology}

\subsection{Experimental Apparatus and Instrumentation}

The experimental set-up presented in Figure 1 consists of a steel chamber where a hydraulic control unit drives a piston generating a periodic bi-directional airflow. Flow speed can be controlled by adjusting amplitude and period of piston motion. A linear potentiometer measures the piston position during its motion and is used for feedback control in the hydraulic unit.

A typical sinusoidal piston motion is reported in Figure 2 in terms of non-dimensional period $t / T$ and non-dimensional position $Z^{*}$, defined as follows:

$$
Z^{*}=\frac{z-z_{\min }}{z_{\max }-z_{\min }}
$$

In the top section of the chamber, a high solidity Wells turbine is housed and drives an induction motor controlled by an inverter with encoder feedback. The turbine's dimensions and test operating parameters are reported in Table 1.

Torque measurements are obtained by a torque meter placed in between the turbine shaft and the motor, while rotational speed is measured with the built-in optical encoder. Torque meter operating range is equal to $\pm 2 \mathrm{Nm}$ with a maximum measurement error of $0.1 \%$ of full scale.

Flow characterization is conducted by means of pressure measurements. Wall pressure taps have been placed at the ambient and piston sides of the rig, $7.5 \mathrm{~mm}$ away from the turbine, while a 3-tubes probe has been used to determine flow characteristics along the blade 


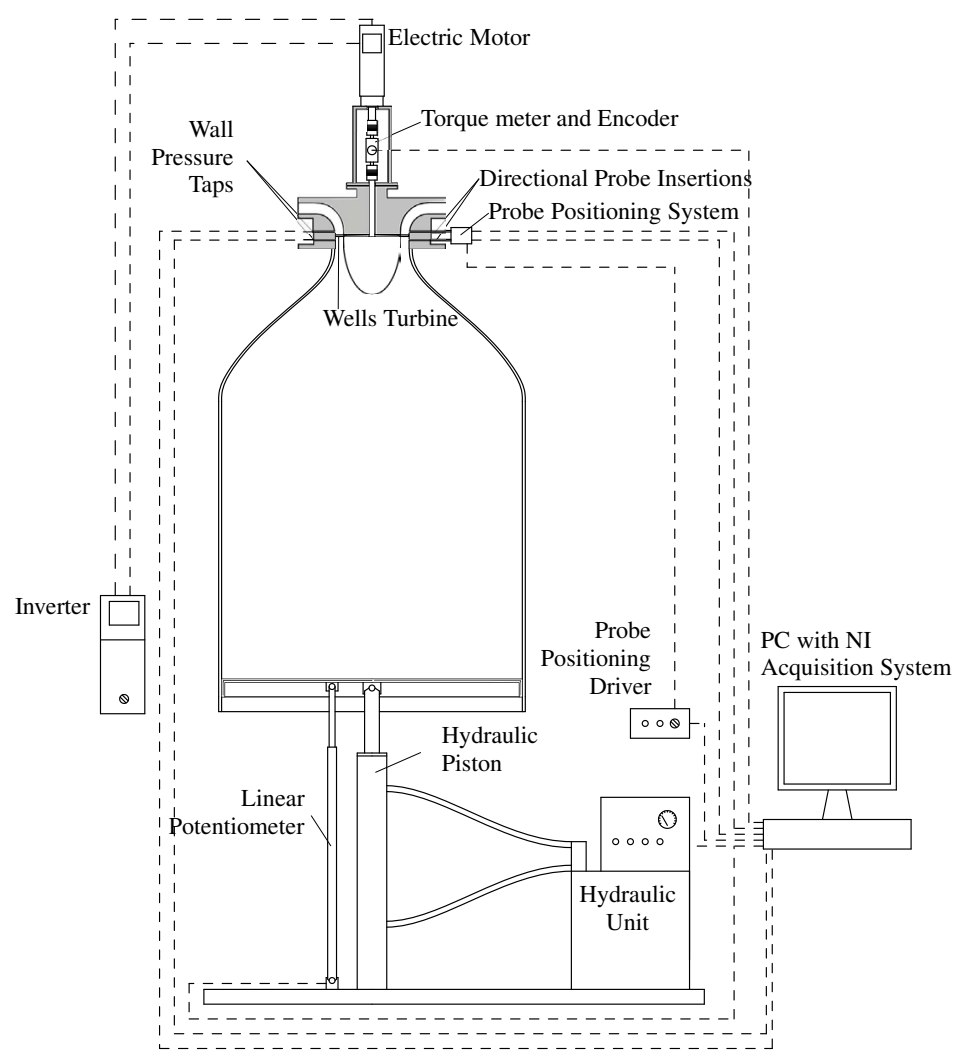

Figure 1: Simplified scheme of the experimental rig.

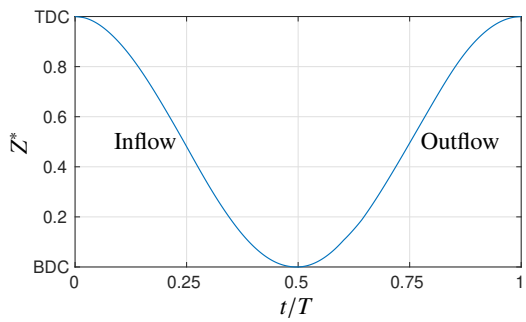

Figure 2: Non-dimensional piston motion as fixed in experiments.

span, both at turbine inlet and outlet. The flow reconstruction has been conducted under the assumption of two-dimensional flow field, hence neglecting the radial velocity, small when compared to axial and tangential components, especially upstream of the rotor [10]. This allows the use of a miniaturized 3-tubes probe able to perform a fine scan of the channel. The probe position has been varied by using a motorized system in order to carry out measurements within the angular calibration range of the probe. Pressure transducers' ranges have been selected depending on the maximum pressure expected at a given location, in order to avoid large errors. 
Table 1: Wells turbine data

\begin{tabular}{ll}
\hline Rotor Tip Diameter [mm] & 250 \\
Rotor Hub Diameter [mm] & 190 \\
Tip Clearance $[\mathrm{mm}]$ & 1 \\
Chord Length [mm] & 36 \\
Sweep Ratio & $0.417(15 / 36)$ \\
Number of Blades & 14 \\
Airfoil Profile & NACA0015 \\
Solidity & 0.729 \\
Rotational Speed $[\mathrm{rpm}]$ & 3,600 \\
$\begin{array}{l}\text { Operating Frequency }\left[\mathrm{s}^{-1}\right] \\
\text { Reynolds' number of }\end{array}$ & $1 / 9$ \\
the outlet flow & $\approx 1.30 \times 10^{5}$ \\
(based on blade chord) & \\
\hline
\end{tabular}

Probe positioning and data acquisition have been performed with a PC equipped with a National Instruments multifunction DAQ-board controlled with a MatLab software.

Investigations have been conducted by setting up a sinusoidal piston motion with a period of $9 \mathrm{~s}$ and an amplitude of $900 \mathrm{~mm}$. The turbine's rotational speed has been set at 3,600 rpm and kept almost constant by the inverter's controller. Under these conditions, the turbine works near the stall region at the maximum flow coefficient, where the maximum output torque is expected. At least 5 piston periods have been acquired for every probe position along the blade span, in order to obtain a periodic mean distribution of the non-stationary flow variables after a phase-locked averaging processing of the signals.

\subsection{Aerodynamic Probe}

The aerodynamic probe used for local flow reconstruction is the miniaturized "Cobra" probe shown in Figure 3. It has been placed $15 \mathrm{~mm}$ away from the turbine, both at its inlet and outlet side. The probe's head has a maximum dimension in the radial direction of about 1 $\mathrm{mm}$ that allows a fine resolution of the flow near the walls, reducing flow distortion around it, in the small channel housing the turbine.

The probe has been used in "non-nulling" mode within its angular calibration range, in order to determine the flow angle on the tangential plane, total and static pressures. The probe's approximate orientations with respect to the absolute flow field both at the inlet and the outlet of the turbine are represented in Figure 3. Probe calibration has been conducted in a subsonic wind tunnel by relating the pressure measured by the front tap, $p_{f}$, to the total pressure, the differential pressure between left and right taps, $p_{l}-p_{r}$, to the yaw angle and the mean pressure for left and right taps, $\left(p_{l}+p_{r}\right) / 2$, to the static pressure. Figure 4 shows the calibration coefficients of the probe.

The yaw coefficient $K_{Y}$ has been non-dimensionalized with respect to the dynamic pressure of the probe, i.e. $p_{f}-\left(p_{l}+p_{r}\right) / 2$, while total and static pressure coefficients $K_{T}$ and $K_{S}$, are non-dimensionalized with respect to the true dynamic pressure of the flow. Figures 4 (a) and (b) show that the three calibration coefficients are symmetric inside the wide yaw range of calibration.

The probe's capability to reconstruct bi-dimensional flow fields has been verified in a wind tunnel. Measurements have shown an uncertainty in total and static pressure of $\pm 1.5 \%$ of the dynamic pressure with a maximum error in flow angle detection of \pm 0.5 degrees. 


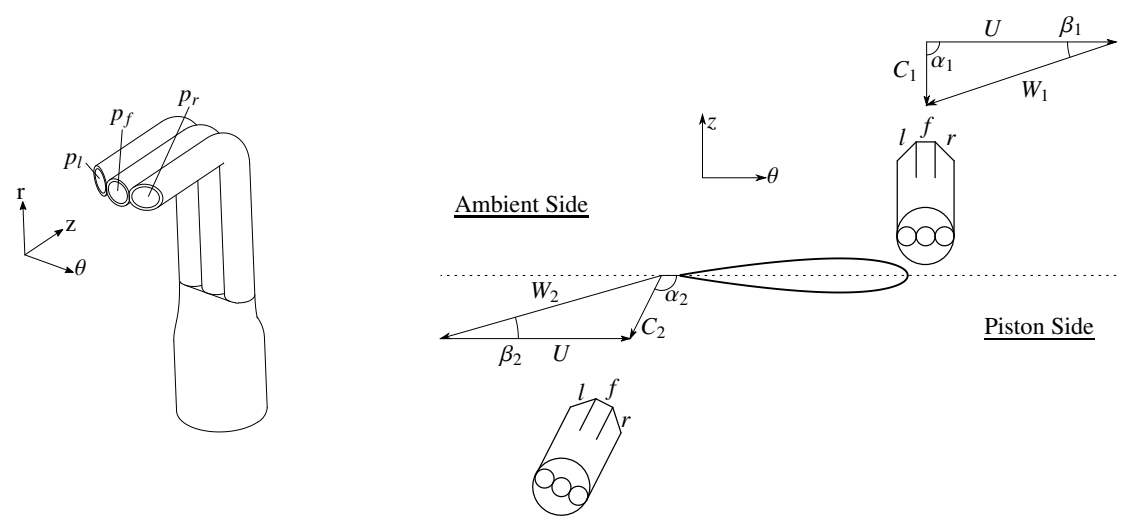

Figure 3: Probe configuration and positioning upstream and downstream of the rotor during the inflow phase.

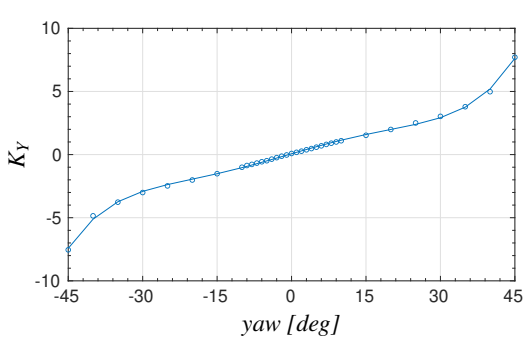

(a) Yaw coefficient.
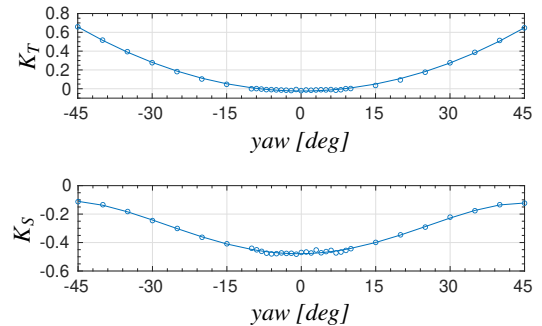

(b) Total, $K_{T}$, and static, $K_{S}$, pressure coefficient.

Figure 4: Non-dimensional calibration coefficients as a function of the yaw range for the "Cobra" probe.

\section{Results and discussion}

\subsection{Inlet Flow}

Figure 5 shows velocity components and flow angles, (a) and (b), respectively, evaluated at the blade midspan during the inflow phase.

The axial velocity component is well resolved during all the inflow phase. As expected, a parabolic distribution of the axial velocity is obtained and the tangential velocity component presents a small value due to the potential effect induced by the rotor. The absolute inlet angle, $\alpha_{1}$, appears constant during the entire phase while the relative flow angle, $\beta_{1}$, i.e. the angle of incidence, varies proportionally to the axial velocity component as the flow rate changes.

The substantially axial nature of the inlet flow allows its characterization by measuring the total pressure along the blade span with a very small flattened probe (nose radial dimension equal to $0.35 \mathrm{~mm}$ ). By combining this measure with the static pressure measurement at the wall, the boundary layer profile can be obtained. In Figure 6, the absolute velocity nondimensionalized with the undisturbed mean velocity (outside the boundary layer) is shown, as a function of the non-dimensional radial position $r^{*}$ : 


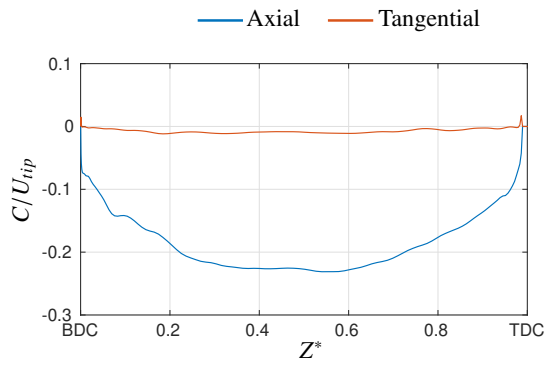

(a) Velocity components.
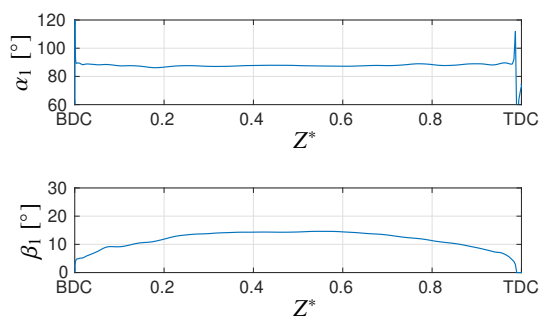

(b) Flow angles.

Figure 5: Velocity components and flow angles measured at blade midspan as a function of the non-dimensional piston position, at the turbine inlet.

$$
r^{*}=\frac{r-r_{h u b}}{r_{t i p}-r_{h u b}}
$$

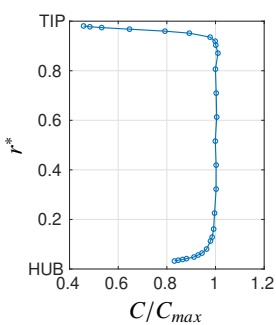

Figure 6: Non-dimensional velocity distribution along the blade span at the maximum flow coefficient.

The boundary layer appears to be very thin both near tip and hub walls and most of the channel height is subjected to an uniform flow.

Non-dimensional velocity components evaluated with the 3-tubes probe are reported in Figure 7 (a) as a function of the spanwise position, for the maximum flow rate. The presence of the $90^{\circ}$ bend at inlet section, see Figure 1, determines a positive gradient for the axial velocity along the blade span, with the velocity reaching its maximum absolute value in the tip region (where the radius of curvature is the lowest). No significant tangential velocity component has been detected along the channel height.

More interesting are the flow angles distributions along the blade span, as reported in Figure 7 (b). Both the absolute and relative inlet angle, $\alpha_{1}$ and $\beta_{1}$, are almost constant along the blade span. While this is natural for the absolute inlet angle $\alpha_{1}$, due to the the fact that the flow is axially guided by the inlet duct, the relative flow angle $\beta_{1}$ is approximately constant due to the presence of the inlet bend, which causes an increase in the axial velocity component that compensates the rise in blade speed at larger radial positions.

\subsection{Outlet Flow}

Figure 8 (a) and (b) report velocity components and flow angles, respectively, measured at mid-channel height, as a function of the non-dimensional piston position $Z^{*}$. 


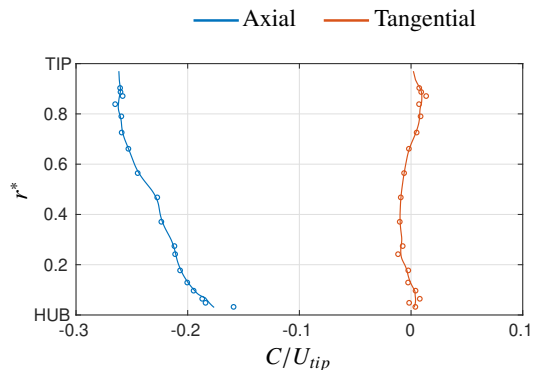

(a) Non-dimensional velocity components.
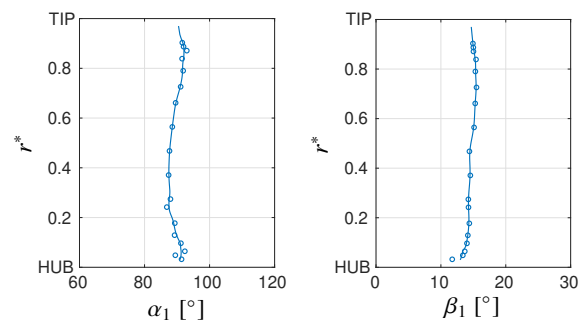

(b) Flow angles.

Figure 7: Non-dimensional velocity components and flow angles along the blade span at the maximum flow coefficient at the turbine inlet.

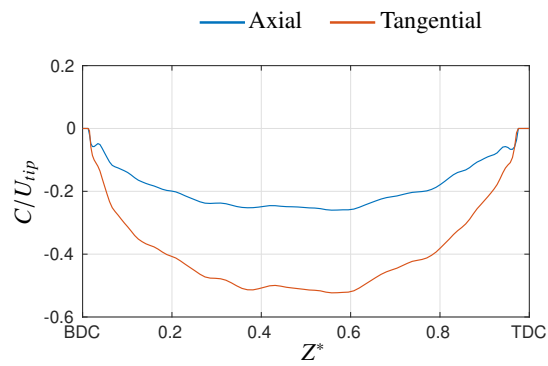

(a) Non-dimensional velocity components.
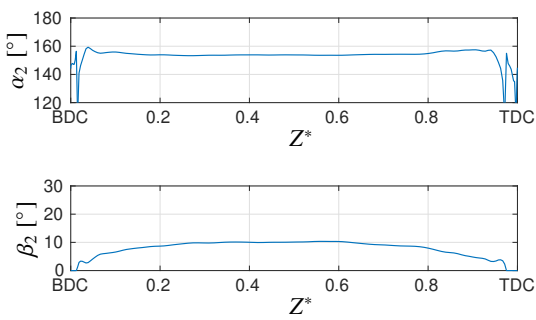

(b) Flow angles.

Figure 8: Velocity components and flow angles measured at blade midspan as a function of the non-dimensional piston position, at the turbine outlet.

The tangential velocity component remains significant during the entire phase, in agreement to the Euler equation for the work exchanged by the rotor, while the axial component is similar in trend and intensity to the one measured at the rotor inlet, as the flow rate through the turbine is conserved. The relative flow angle $\beta_{2}$, i.e. the angle of deviation, shows a trend similar to $\beta_{1}$ in the inlet phase, varying from zero to a maximum of $10^{\circ}$ when the incidence angle is the largest. The distribution of the absolute flow angle $\alpha_{2}$ shows an almost constant value during the whole phase.

Velocity components and flow angles along the blade span are reported in Figure 9 (a) and (b), respectively.

It can be observed that the effect of the high turbine solidity determines a significant blockage, which is the cause of the low values of velocity and flow angles. Near the hub region, for about $20 \%$ of the blade span, the flow blockage is pronounced and the velocity is almost oriented tangentially, in the direction opposite to the blade speed $U$. In the midspan region, as the vane passage grows, a higher axial component is measured and the flow angle shows an increase of the flow deviation; the tangential velocity also grows with the radius and reaches its maximum intensity around midspan. In the tip region, the combined effects of the higher axial velocity component at the inlet, the lower solidity and the tip gap are responsible for the large axial velocity at the rotor exit. 


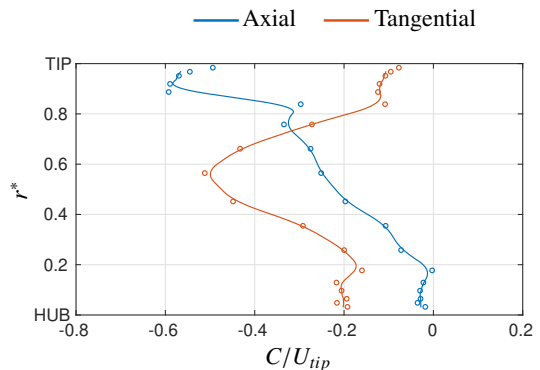

(a) Non-dimensional velocity components.
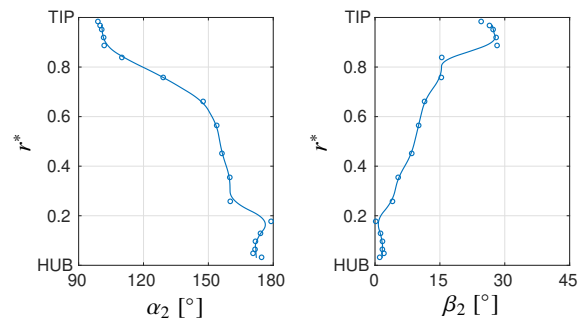

(b) Flow angles.

Figure 9: Non-dimensional velocity components and flow angles along the blade span at the maximum flow coefficient, at the turbine outlet.

\subsection{Turbine Performance}

Performance of Wells turbines is defined in terms of flow coefficient $\phi$, torque coefficient $T^{*}$ and head coefficient $P^{*}$ according to Equation (3).

$$
\phi=\frac{C_{z}}{\omega r_{t i p}} \quad T^{*}=\frac{T}{\rho \omega^{2} r_{t i p}^{5}} \quad P^{*}=\frac{\Delta P}{\rho \omega^{2} r_{t i p}^{2}}
$$

where $C_{z}$ is the mean axial velocity component measured at rotor inlet, $\omega$ is the rotor angular velocity, $r_{t i p}$ is the tip radius of the rotor, $T$ is the torque measured at turbine's shaft and $\Delta P$ is the total pressure drop across the turbine.

As in Wells turbines the exhaust kinetic energy is entirely wasted $[8,16,17]$, the total-tostatic efficiency is considered:

$$
\eta_{t s}=\frac{T \omega}{\left(P_{1}-p_{2}\right) \cdot Q}
$$

where the available power is defined as the product of volumetric flow rate $Q$ and the totalto-static pressure drop across the rotor $P_{1}-p_{2}$. The volumetric flow rate has been calculated using the axial velocity distribution along the blade span, measured with the aerodynamic probe. Although this method is more complicated than the estimation based on piston speed, it is necessary to eliminate the delay [18] between the piston velocity and flow speed at the turbine inlet induced by the presence of the chamber volume.

Performance parameters are summarized in Figure 10 as a function of the flow coefficient $\phi$.

The selected operating conditions allow to avoid turbine stall, as shown by the nondimensional torque in Figure 10 (a). The torque coefficient rises monotonically with the flow coefficient, and there is a range, i.e for $\phi=0 \div 0.1$, where the turbine does not produce an useful torque but it absorbs energy from the line to keep its rotating speed constant. This same region is well identifiable in the turbine efficiency plot, Figures 10 (c), which presents a negative value. The working range, $\phi=0.1 \div 0.23$, is characterized by a positive efficiency that reaches its maximum value for $\phi \approx 0.2$, in line with the results reported in [10] for the same turbine in term of total-to-total efficiency.

Performance parameters defined in Equations (3) and (4) are also evaluated along the blade span for three different flow conditions, i.e. $\phi=0.15$ in the accelerating phase, the maximum flow rate $\phi=0.23$ and $\phi=0.15$ in the decelerating phase, in Figure 11 . 


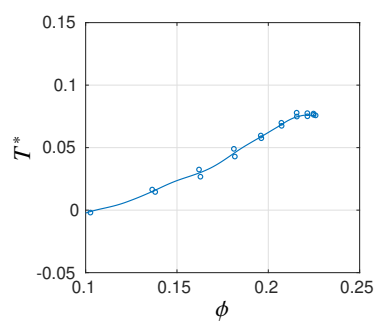

(a) Torque coefficient.

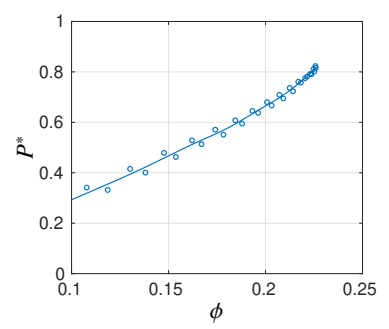

(b) Head coefficient.

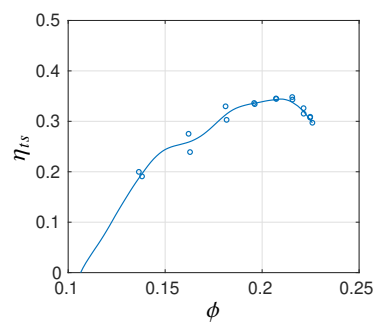

(c) Total-to-static efficiency.

Figure 10: Wells turbine performance parameters as a function of the flow coefficient.

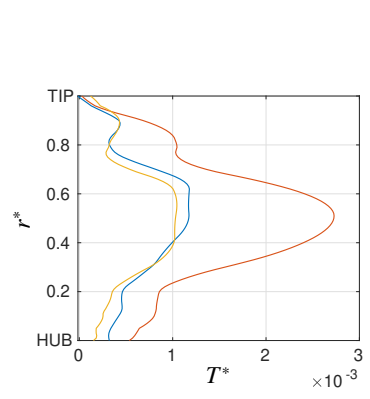

(a) Torque coefficient.

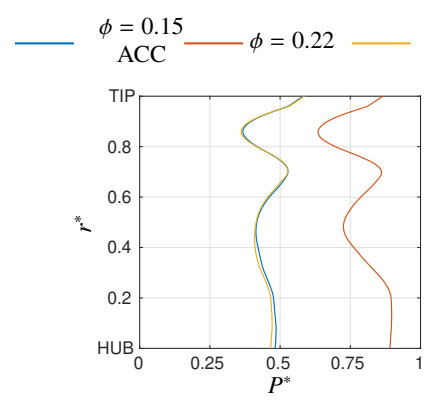

(b) Head coefficient.

$$
\begin{aligned}
& \phi=0.15 \\
& \text { DEC }
\end{aligned}
$$

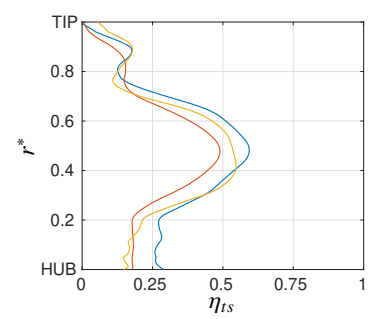

(c) Total-to-static efficiency.

Figure 11: Distributions of the non-dimensional performance parameters as a function of blade span for different flow conditions.

Radial distributions reported in Figure 11 highlight the aerodynamic behavior of the blade row. In this case, the performance parameters have been based on local flow measurements obtained from the aerodynamic probe, by calculating the aerodynamic torque $T_{a d}(r)$ using the Euler equation:

$$
T_{a d}=\dot{m} r\left(C_{1 \theta}-C_{2 \theta}\right)
$$

where subscript $\theta$ denotes the tangential component of inlet and outlet absolute velocities and $\dot{m}$ is the local mass flow rate. Then, the torque coefficient $T^{*}$ represents the nondimensionalized aerodynamic torque produced by the rotor without considering windage and inertial contribution. Its trend depends on velocities distributions in Figures 7 and 9: a higher torque in the midspan region and lower torque values in tip and hub regions. The head coefficient $P^{*}$, that represents the blade load is higher for the largest values of the flow coefficient $\phi$, i.e. for the highest volumetric flow rates. The efficiency $\eta_{t s}$ of pneumatic energy conversion of the flow into work shows higher values in the midspan region and reduces in the tip region, similarly to the torque coefficient. Radial distributions in Figure 11 present only differences in values while their trend remain substantially unchanged for the selected flow coefficients $\phi$.

These results are useful to identify the regions of the blade where action needs to be taken to improve its aerodynamic behavior. In particular, in the hub region, the blockage effect is responsible for the lower measured performance. A reduction in rotor solidity at the blade root is necessary to mitigate this effect. Performance in the tip region are mainly affected by 
the tip leakage that, due to the high hub-to-tip ratio of the blade, strongly affects the flow also in the midspan region.

\section{Conclusions}

This paper investigates the local flow field at the inlet and outlet of a Wells turbine submitted to a non-stationary airflow, as it happens inside an Oscillating Water Column system. The experimental investigations have been carried out in a laboratory set-up by using aerodynamic pressure probes assuming a bi-dimensional flow. Non-dimensional parameters have been used to characterize global and local performance of the tested rotor. The main results of this preliminary work can be summarized as follows:

1. The inlet flow from the ambient is directed by the bent duct which determines a static pressure gradient along the blade span. An almost constant angle of incidence at different radial positions is obtained; this behavior reduces the blockage effect induced by the high solidity in the hub region.

2. The flow at the exit of the rotor is characterized by the strong blockage effect at the blade root, which reduces the velocity component, and by the leakage due to the tip gap, which affects a relatively large portion of the blade height.

3. As a result of the local measurements, the performance parameters show large variations along the blade span with the best values only in the midspan region.

Local performance analyses are useful to lead the aerodynamic optimization of the blade profile by varying its geometry. In particular, simple improvements could be obtained by reducing the blade chord in the hub region and by reducing the tip gap.

Although this paper presents results only for the inflow phase of the piston motion, i.e. where the airflow is coming from the atmosphere to the piston chamber, and the input geometry is different during the opposite phase, no significant differences are expected both for local and global performance. This is confirmed by previous analyses [9, 10], where an absolute inlet flow angle different from $90^{\circ}$ has been observed during the outflow phase.

\section{References}

[1] R. Pelc, R. Fujita, Marine Policy 26, 471 (2002)

[2] A. Falcão, Renewable and Sustainable Energy Reviews 14, 899 (2010)

[3] A. Falcão, J. Henriques, Renewable Energy 85, 1391 (2016)

[4] A. Wells, Fluid Driven Rotary Transducer - BR. Pat. 1595700 (1976)

[5] T. Dhanasekaran, M. Govardhan, Renewable Energy 30, 2129 (2005)

[6] M. Torresi, S. Camporeale, P. Strippoli, G. Pascazio, Renewable Energy 33, 735 (2008)

[7] T. Ghisu, P. Puddu, F. Cambuli, Proceedings of the Institution of Mechanical Engineers, Part A: Journal of Power and Energy 231, 197 (2017)

[8] F. Licheri, F. Cambuli, P. Puddu, T. Ghisu, Journal of Fluids Engineering 143 (2021)

[9] P. Puddu, M. Paderi, C. Manca, Energy Procedia 45, 278 (2014)

[10] M. Paderi, P. Puddu, Renewable Energy 57, 570 (2013)

[11] R. Curran, L.M.C. Gato, Proceedings of the Institution of Mechanical Engineers, Part A: Journal of Power and Energy 211, 133 (1997)

[12] A. Thakker, R. Abdulhadi, Renewable Energy 33, 2467 (2008)

[13] S. Raghunathan, C. Tan, Journal of energy 7, 226 (1983) 
[14] M. Torresi, M. Stefanizzi, F. Fornarelli, L. Gurnari, P.G.F. Filianoti, S.M. Camporeale, AIP Conference Proceedings 2191, 020149 (2019)

[15] M. Takao, T. Setoguchi, S. Nagata, K. Toyota, 6, 625 (2008)

[16] S. Raghunathan, C.P. Tan, O.O. Ombaka, Aeronautical Journal 89, 369 (1985)

[17] L.M.C. Gato, A.F.d.O. Falcão, Journal of Engineering for Gas Turbines and Power 106, 628 (1984)

[18] T. Ghisu, F. Cambuli, P. Puddu, I. Virdis, M. Carta, F. Licheri, Applied Ocean Research 94, 101994 (2020) 